\title{
La gouvernance d'entreprise au Canada : un domaine en transition
}

\author{
Imen Latrous ${ }^{a}$, Marc-André Morency ${ }^{b}$, Salmata Ouedraogo ${ }^{c}$, Jeanne Simard ${ }^{d, 1}$
}

RÉSUMÉ. De nombreux chercheurs ont mis en évidence les aspects et conséquences discutables de certaines conceptions financières ou théories de l'organisation. C'est le cas de la théorie de l'agence, conception particulièrement influente depuis une quarantaine d'années, qui a pour effet de justifier une gouvernance de l'entreprise vouée à maximiser la valeur aux actionnaires au détriment des autres parties prenantes. Cette idéologie de gouvernance justifie de rémunérer les managers, présumés négliger ordinairement les détenteurs d'actions, avec des stock-options, des salaires démesurés. Ce primat accordé à la valeur à court terme des actions relève d'une vision dans laquelle les raisons financières se voient attribuer un rôle prééminent dans la détermination des objectifs et des moyens d'action, de régulation et de dérégulation des entreprises. Cet article se propose de rappeler les éléments centraux de ce modèle de gouvernance et de voir quelles critiques lui sont adressées par des disciplines aussi diverses que l'économie, la finance, le droit et la sociologie.

ABSTRACT. Many researchers have highlighted the questionable aspects and consequences of certain financial concepts or theories of the organization. This is the case of agency theory, which has had an anormous influence over the past forty years; it justifies a form of corporate governance aimed at maximizing shareholder value to the detriment of other stakeholders. This ideology of governance justifies remunerating managers, presumably neglecting the shareholders, with stock options and excessive wages. This primacy given to the shortterm value of equities comes from a vision in which financial reasons are given a pre-eminent role in determining the objectives and means of action, regulation and deregulation of companies. This article aims to recall the central elements of this model of governance and the criticisms addressed to it by disciplines as diverse as economics, finance, law and sociology.

La réalité mondiale se présente actuellement comme un nœud de forces très contradictoires. On observe, d'une part, la mise en place d'outils normatifs et éthiques de responsabilisation rétrospective et prospective (comme le principe de précaution) visant à ramener la confiance du public envers ses institutions, à protéger les personnes les plus vulnérables et à instaurer un véritable développement collectif. À l'opposé, certaines théories de l'économie et du management justifient la recherche d'avantages individuels sous différentes formes, sans égard aux conséquences à long terme pour les salariés, l'environnement, la société et les générations futures. La croissance des inégalités que l'on observe depuis les années 1970 traduit le fait que de nombreux dirigeants, gestionnaires, administrateurs d'organisations publiques et privées, soit abusent de leur position, soit sont pris en otage par les règles qui ont cours. Les crises financières récurrentes sont un révélateur des excès qui en résultent (Stiglitz, 2015).

Le marché financier a ainsi été ébranlé par une série d'effondrements de géants qu'on croyait indestructibles comme Enron, WorldCom, Nortel, Lehman Brothers, LTCM, Barings, etc. Par quelles voies en est-on arrivé à de telles catastrophes dans le monde des grandes entreprises? Comment expliquer que l'on connaisse des situations aussi "paradoxantes " pour la gouvernance des organisations? (Gaulejac

\footnotetext{
a Professeure, D. Sc., Université du Québec à Chicoutimi

b Professeur retraité, sociologue, Université du Québec à Chicoutimi

c Professeure, Ph. D., Université du Québec à Chicoutimi

d Professeure, LL. D., avocate, F. Adm.A., Université du Québec à Chicoutimi
} 
et Hanique, 2015) L'analyse permet de comprendre qu'il y a là bien plus qu'une question d'avidité, même si certains traits de caractère se trouvent particulièrement valorisés dans le système économique actuel. Des causes structurelles sont à l'origine de cette confrontation de valeurs et de comportements, causes qui vont bien au-delà de la psyché et de l'éthique des personnes et c'est dans le domaine des institutions en l'occurrence qu'il convient de cerner de plus près les processus à l'origine de ces situations (Bardelli, 2012; Boyer, 2011, 2015).

De nombreux chercheurs ont en effet mis en évidence les aspects et conséquences discutables de certaines conceptions financières ou théories de l'organisation. C'est le cas de la théorie de l'agence, conception particulièrement influente depuis une quarantaine d'années, qui a pour effet de justifier une gouvernance de l'entreprise vouée à maximiser la valeur aux actionnaires au détriment des autres parties prenantes. Cette idéologie de gouvernance justifie de rémunérer les managers, présumés négliger ordinairement les détenteurs d'actions, avec des stock-options, des salaires démesurés. Ce primat accordé à la valeur à court terme des actions relève d'une vision dans laquelle les raisons financières se voient attribuer un rôle prééminent dans la détermination des objectifs et des moyens d'action, de régulation et de dérégulation des entreprises. Cet article se propose, 1) de rappeler les éléments centraux de ce modèle de gouvernance et, 2) de voir quelles critiques lui sont adressées par des disciplines aussi diverses que l'économie, la finance, la sociologie et le droit.

\section{La théorie de l'agence : contexte d'origine et description}

Avant d'aborder plus en détail cette théorie de l'agence qui oriente de façon importante la gouvernance des organisations, il importe d'effectuer un retour sur les aspects contextuels de son émergence au cours des années 1970. Propulsée par une soudaine envolée des prix du pétrole consécutive à une décision de l'Organisation des pays exportateurs de pétrole (OPEP), l'inflation ravage les économies qui entrent simultanément en stagnation économique, une situation sans précédent devant laquelle la théorie économique standard reste sans moyens. Cette « stagflation » entraîne une crise du dollar américain qui cesse en 1973, sous Nixon, de maintenir son lien avec l'or. L’approche néokeynésienne préconisant de jumeler l'action de l'État à celle de l'entreprise privée, dans le dessein d'équilibrer les rapports entre le capital et le travail, doctrine qui a dominé l'après-guerre, période appelée « les Trente Glorieuses ", semble en panne. Le discours ultralibéral de Friedrich August von Hayek et des membres de la Société du Mont-Pèlerin trouve dans ce contexte un écho plus favorable. L'idéologie monétariste de l'économiste Milton Friedman, laquelle réduit le rôle de l'État dans le contrôle de la monnaie, va alors trouver un premier champ d'expérimentation au Chili qui voit Augusto Pinochet liquider le gouvernement Allende, et y faire l'expérience des théories de l'École de Chicago.

La crise qui frappe l'économie mondiale permet ainsi aux théoriciens et politiciens conservateurs de limiter le rôle social de l'État, et d'enclencher une vague de privatisation de services et d'entreprises publics, jugés inefficaces et coûteux. Margaret Thatcher, première ministre de Grande-Bretagne, dont les slogans « There is no alternative " (TINA), "There is no such thing as society ", sont bien connus, entraîne les partis conservateurs de l'Occident, les chefs d'État Ronald Reagan (É.-U.) et Brian Mulroney (Canada), dans la déconstruction de l'État néokeynésien. Sont également abattues des murailles de Chine qui servaient depuis le New Deal à contrôler le domaine financier (Truffaud, 2017), séparant les activités de dépôt, d'investissement, et d'assurance. Ces décisions vont permettre aux banques d'élargir leur champ d'activité, de les affranchir du calcul des risques et de la régulation, de jouer pleinement sur les franges de l'aléa moral, voire construire de nouvelles variantes des schémas de Ponzi. La financiarisation de l'économie, « le tout acquis aux exigences de la finance », est en marche. Le contrôle de la création monétaire et du crédit permet aux banquiers de réguler l'État et de l'endetter, de soumettre les entreprises à une scrutation permanente de leur gouvernance, de leur profitabilité. Cette reconstruction du domaine de l'État et de l'économie frappe très largement.

La majorité des gouvernements adopte des mesures favorisant la retraite par capitalisation, le recours aux assurances privées, le développement de l'actionnariat des salariés, la privatisation des services publics, la tolérance vis-à-vis des dispositifs de défiscalisation, l'acceptation de normes comptables internationales conçues par des organismes privés (Gaulejac et Hanique, 2015, p. 59). 
En synthèse, on assiste à « un retour au raisonnement libéral micro-économique fondé sur la maximisation du profit privé et la régulation par le marché » (Gomez, 2009, p. 373). Dans un tel contexte, les mots d'ordre sont : libération des acteurs financiers, rôle normatif des théories de la propriété privée, le marché comme principal régulateur de l'économie, liberté individuelle d'entreprendre à tous les niveaux de la société. Comme la situation économique en général ne permet pas de produire facilement les capitaux, de prêter et d'emprunter, on se tourne vers l'entrepreneur dont il faut libérer les ailes, et vers un actionnariat qu'il faut séduire par des rendements élevés. Une telle posture entraîne une massification de l'actionnariat par le truchement de fonds de retraite capitalisés. La capitalisation boursière mondiale explose. En effet, selon Bloomberg, la capitalisation boursière mondiale à la fin de 2013 était de quelque 61975 milliards de dollars (Fay et Boisseau, 2014). Dans ce contexte, la maximation de la richesse des actionnaires constitue le principal objectif financier des entreprises. Dans la foulée de cette « réorientation » du raisonnement économique qui passe de la macro vers le micro, on repense la conception de l'entreprise de même que la façon de la gouverner (corporate governance).

L'organisation elle-même devient une liquidité, à l'image de n'importe quel produit financier. Son statut d'institution économique, d'acteur majeur de la production de biens et de services, d'élément central dans la construction de la société se liquéfie dans une finalité qui se déplace du registre productif au registre financier. $[\ldots]$

Le capital n'est plus un moyen parmi d'autres pour permettre à l'entreprise de produire, c'est l'entreprise qui devient un capital dont les investisseurs cherchent à maximiser les performances (Gaulejac et Hanique, 2015, p. 61; Orléan, 1999).

Pour Milton Friedman (1970) et l'École de Chicago, la seule responsabilité sociale de l'entreprise est de faire du profit, même si des actions de philanthropie ou de mécénat peuvent à l'occasion être envisagées ${ }^{2}$. La performance optimale de l'entreprise requiert d'amener les actionnaires à influer sur cette dernière pour en optimiser la gestion, et ce, en minimisant l'influence des autres parties prenantes. On mise sur le fait que « le propriétaire capitaliste est le meilleur garant de la bonne gestion de l'entreprise puisqu'il en va de sa propriété privée » (Alchian et Demsetz, 1973, cité par Gomez, p. 373).

Parmi les théories de référence propageant une gouvernance d'entreprise dominée par la rhétorique financière se trouve la «théorie » de l'agence ${ }^{3}$. À l'origine, cette théorie qui « a atteint un statut prédominant au sein des théories financières et organisationnelles » (Boncori et Mahieux, 2012, p. 132) se présente comme le résultat d'un ambitieux projet de l'Université de Rochester et plus particulièrement des professeurs Michael C. Jensen et William H. Meckling (1976). Le projet avait pour objectif de construire une théorie du comportement humain au sein des organisations en partant de l'hypothèse de la rationalité des acteurs économiques. En effet, dans leur article «La nature de l'homme », Jensen et Meckling (1994) proposent un modèle du comportement humain (REMM : Resourceful, Evaluative, Maximizing Model) reposant sur des postulats selon lesquels « les individus se préoccupent de tout ce qui est source d'utilité, ils sont insatiables, cherchant, sous des contraintes cognitives, à maximiser leurs choix pécuniaires ou non, créatifs, et savent s'adapter » (Charreaux, 2005, p. 6-7). Dans cette logique, les individus sont perçus comme des opportunistes qui savent créer ou saisir des occasions en réalisant un arbitrage, et ce, pour leur propre intérêt et au détriment des autres (self-interested).

L'entreprise n'a pas d'existence indépendante; elle est représentée comme un regroupement de parties prenantes uni par un faisceau ou nœud de contrats (écrits ou non écrits).

Dans le cadre de cette théorie, la firme n'est ni une entité ni une chose capable d'être possédée. Elle est simplement une fiction légale qui comprend un jeu de relations contractuelles qui établissent des droits et des obligations (Rousseau, 2007).

Chaque partie prenante se voit imposer par contrat des règles du jeu (droits et obligations) différentes, car elles n'ont pas toutes le même statut. Les actionnaires n'ont pas droit automatiquement ou statutairement à une rémunération, à un rendement fixe garanti, comme les autres parties prenantes. Ils assument une partie des risques résiduels, c'est-àdire qu'« ils obtiennent ce qui reste (le cas échéant) après que les revendications contractuelles de tout le monde aient été satisfaites » (Maitland, 1994, p. 449 cité par Hasnas, 2012, p. 51 [notre 
traduction]). Les actionnaires deviennent une sorte de caution pour les autres parties prenantes (Hasnas, 2012). À titre d'exemple, les salariés reçoivent leur rémunération de façon prioritaire ${ }^{4}$, puis viennent les fournisseurs, les municipalités, etc. Si l'entreprise fait suffisamment de profit, les actionnaires peuvent percevoir des dividendes sur leur investissement; la valeur de leurs actions pourra monter. Le conseil d'administration prend la décision de distribuer les dividendes de façon discrétionnaire en fonction des conditions du marché et compte tenu de la situation financière de la société par actions ${ }^{5}$. Le conseil d'administration peut aussi décider de constituer un fonds de réserve. Pour les tenants de cette vision financière de l'entreprise, divers risques rendent les actionnaires plus vulnérables à une mauvaise gestion, à une négligence de la part des dirigeants. La ligne est mince permettant de prétendre que les actionnaires sont les mieux placés pour exiger la maximisation du profit et pour détenir le pouvoir de contrôle ultime. Ils sont amenés ainsi en première ligne pour prévenir les erreurs de gestion (Gomez, 2009 citant Hart et Moore, 1990; Jensen, 2000).

Dans cet esprit, il fut considéré légitime que les règles de gouvernance organisationnelle s'orientent vers une obligation fiduciaire prépondérante des dirigeants de l'entreprise envers les actionnaires individuels et surtout institutionnels (les régimes de retraite, les fonds de placement, etc. $)^{6}$. Le dirigeant est alors présenté comme un agent responsable auprès des actionnaires et son objectif principal est de maximiser les retours à ces derniers. La relation d'agence est définie comme "un contrat par lequel une ou plusieurs personnes (le(s) principal(aux)) engage(nt) une autre personne (l'agent) pour agir en leur nom, ce qui implique la délégation à l'agent d'une partie de l'autorité en ce qui concerne le processus de décision » (Jensen et Meckling, 1976, p. 312). Dans cette représentation, il apparait que la relation d'agence ne concerne que deux catégories de personnes, la première étant le principal (les actionnaires), qui délègue un droit décisionnel à l'autre, l'agent (le dirigeant) ${ }^{7}$. La figure 1 résume cette conception :

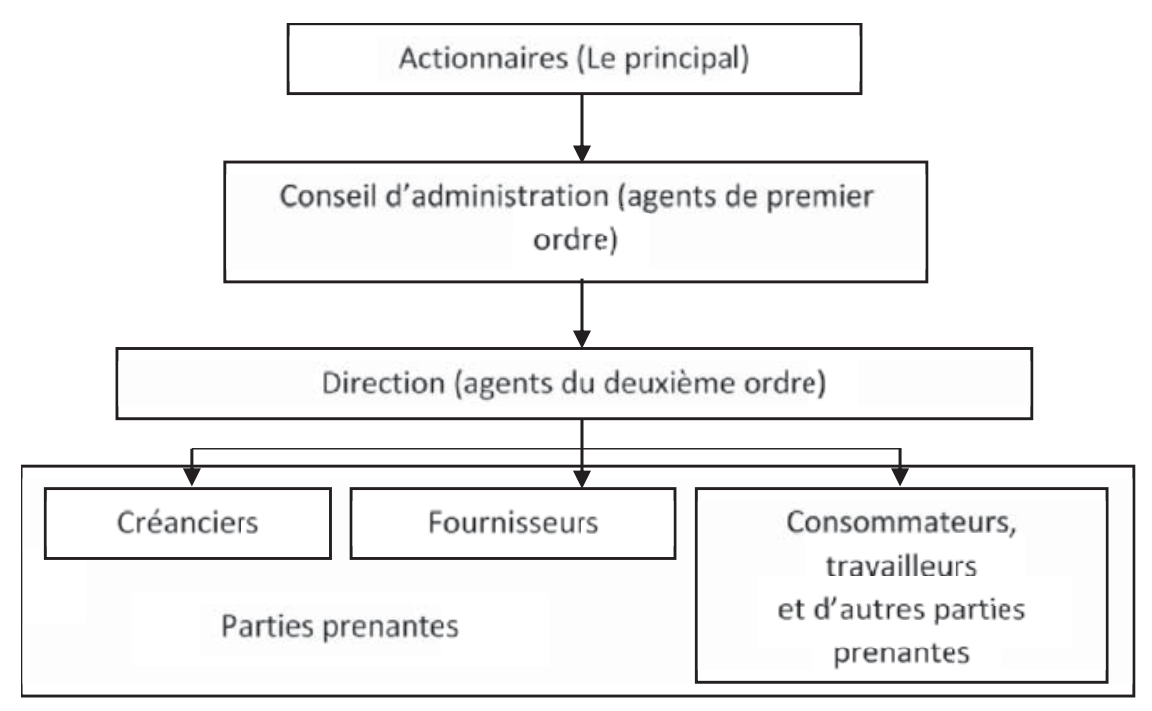

Figure 1 - Le modèle de la primauté des actionnaires (théorie de l'agence) Source : Modèle de Lan et Heracleous (2010), p. 302 (Notre traduction)

Cette relation d'agence entre les dirigeants de l'entreprise et les actionnaires ne va pas sans difficulté, car des conflits entre les parties demeurent toujours possibles. Des conflits dits «d'agence » sont susceptibles d'apparaittre du fait de la divergence des intérêts et de l'asymétrie de l'information entre les parties. En effet, les dirigeants peuvent préférer maximiser leur consommation privée au détriment de la maximisation de la valeur pour les actionnaires. C'est d'autant plus vraisemblable que les dirigeants détiennent des informations privilégiées sur le fonctionnement de l'entreprise.

Dans une relation d'agence, il arrive fréquemment que le principal et l'agent n'aient pas les mêmes intérêts et que l'agent en sache plus sur 
la tâche à accomplir que le principal (sélection adverse). Par conséquent, le contrat qui lie les parties est incomplet de sorte qu'il est impossible pour le principal de s'assurer d'un contrôle parfait de l'agent (Rouleau, 2007, p. 99-100).

Ce qui rend possibles des comportements opportunistes. Pour surveiller les agissements des dirigeants ou pour les inciter à agir dans l'intérêt des actionnaires, il est alors nécessaire d'engager certaines dépenses. La mise en place des techniques de contrôle et d'incitation va de facto engendrer ce qu'on appelle des « coûts d'agence ». Jensen et Meckling (1976) distinguent trois types de coûts (Charreaux, 1997) : les coûts de surveillance (monitoring costs) et d'incitation, les coûts de dédouanement (bonding costs) et les coûts d'opportunité (residual loss). Rouleau spécifie divers coûts engendrés par ces relations de contrôle :

Par exemple, il peut s'agir de systèmes d'évaluation par objectifs ou de systèmes financiers d'intéressement (bonus, participation à la propriété, etc.). Les coûts d'obligation sont les coûts qui permettent au principal de s'assurer que l'agent n'agira pas contre lui. Advenant le cas où l'agent porte préjudice au principal, celui-ci doit s'assurer d'être dédommagé. Ainsi, le coût desassurances en responsabilité civile est un coût d'obligation. Enfin, comme la maximisation effective du bien-être du principal est difficilement atteignable, le principal doit prévoir le coût des écarts entre ses objectifs et les réalisations de l'agent. Par perte résiduelle, on entend donc l'estimation des dépenses pouvant être engendrées par les écarts de comportement de l'agent (Rouleau, 2007 , p. 100).

Plus concrètement, les divergences d'intérêt peuvent être atténuées si l'entreprise met en place un système de rémunération des dirigeants qui pourra comporter la technique d'incitation financière des stock-options ou la détention d'actions par ces derniers. Les stock-options sont des options d'achat d'actions à prix fixe : si, en Bourse, le cours de l'action progresse au-delà du prix convenu pour l'achat (permettant une « levée d'options »), le détenteur de stock-options gagne la différence entre le coût de l'option (le prix fixé) et le cours en Bourse. Cette modalité de gouvernance a justifié de rémunérer les dirigeants, présumés négliger ordinairement les détenteurs d'actions, avec des stock-options et des rémunérations démesurées. Dans ce cas, les intérêts des actionnaires et des dirigeants sont censés converger dans la mesure où ces derniers (dirigeants) détiennent dorénavant des actions de l'entreprise. L'agent a donc intérêt à tout faire pour que le cours progresse le plus possible, et ses intérêts sont alors alignés sur ceux des actionnaires. Sauf que « tout faire » peut signifier aussi truquer les comptes (le cas d'Enron) ou prendre des risques excessifs (le cas de la Société Générale). Au cas où ces risques se réaliseraient, c'est le principal (les actionnaires) qui en supporterait les frais, tandis que l'agent se contenterait de ne rien gagner. Si les choses fonctionnent correctement, l'agent gagne tout comme les actionnaires. L'asymétrie d'information, voire les délits d'initiés, peuvent inciter l'agent à prendre des risques excessifs, sinon catastrophiques pour l'entreprise.

À l'origine, la théorie de l'agence vise les grandes entreprises américaines à l'actionnariat diffus et présentant des conflits d'agence entre dirigeants et actionnaires (Berle et Means, 1932). Cependant, beaucoup de travaux de recherche ont montré que la dispersion de l'actionnariat est un mythe. Dans une série d'articles portant sur le gouvernement des entreprises, La Porta et al. $(1997,1998,1999)$ montrent l'importance de la concentration de l'actionnariat et le conflit opposant les actionnaires de contrôle aux actionnaires minoritaires, et ce, dans de nombreux pays. Dans les entreprises à capital concentré, une autre relation d'agence apparait entre les actionnaires minoritaires (censés agir comme principal) qui de fait délèguent la gestion de leurs capitaux aux actionnaires de contrôle (agent). En raison des divergences d'intérêts entre les actionnaires de contrôle et les actionnaires minoritaires, la relation d'agence entre ces deux groupes d'actionnaires s'accompagne inévitablement de conflits inducteurs de coûts réduisant ainsi les gains potentiels liés à la relation d'agence. Les actionnaires de contrôle sont en effet capables d'orienter la stratégie de l'entreprise conformément à leurs objectifs et extraire ainsi des bénéfices privés au détriment des actionnaires minoritaires (La Porta et al., 1999; Shleifer et Wolfenzon, 2000).

L'influence de la théorie de l'agence va se révéler d'une très grande importance :

La shareholder value va devenir, dans toutes les entreprises cotées en Bourse et contrôlées par 
des investisseurs financiers, l'élément principal qui inspire la " nouvelle gouvernance managériale ». Les petites et moyennes entreprises (PME) vont subir cette évolution de façon indirecte. Elles doivent intégrer ces pratiques et adapter leurs outils de gestion pour répondre aux nouvelles exigences que leur imposent les grandes firmes qui sont leurs plus gros clients (Gaulejac et Hanique, 2015, p. 65).

On a pu souligner que les codes de bonnes pratiques en matière de gouvernance d'entreprise, la composition et les procédures des conseils d'administration ont été influencés par les principes de la théorie de l'agence (Lan et Heracleous, 2010, p. 294). Cette théorie est devenue une pierre angulaire, non seulement de la littérature sur la gouvernance d'entreprise, mais également des politiques et pratiques de gouvernance d'entreprise (Lan et Heracleous, 2010, p. 294).

Il convient de s'interroger : la théorie de l'agence estelle si fiable, si efficace et si incontournable de nos jours? Prend-elle en compte toutes les problématiques organisationnelles au sein d'une organisation?

\section{Les critiques disciplinaires de la théorie de l'agence}

Le caractère réductionniste de la théorie de l'agence qui invite à ramener les objectifs de l'entreprise à dégager de la valeur actionnariale et sa gouvernance à un mécanisme disciplinaire du comportement des dirigeants n'a pas manqué de faire l'objet de contestation. Les critiques portent notamment sur ses préconceptions (Bourdieu, 1980) économiques, financières, sociologiques, juridiques et éthiques. Ces critiques proposent en quelque sorte un projet de refondation de la conception de l'entreprise, du rôle de ses parties prenantes.

\section{1 Économie et finance}

Les sciences économiques et financières ont multiplié les critiques, depuis la fin des années 1980, à l'endroit de la théorie de l'agence. Bien que des conflits d'intérêts découlant des relations entre les actionnaires, le conseil d'administration et les dirigeants soient réels et que les procédés proposés par la théorie de l'agence pour comprendre et gérer ces conflits aient été très populaires, les résultats de plusieurs études empiriques n'ont pas réussi à démontrer leur efficacité économique (Dalton et al.,
2003; Dalton et al., 2007; Bourque, 2014). Les présupposés de la théorie d'agence ont également été jugés non fondés par divers courants critiques, et donc d'un caractère trop négatif pour contribuer à une amélioration de la gouvernance des entreprises. Ces travaux de recherche, en plus de remettre en question les fondamentaux de la théorie de l'agence, ont fait émerger de nouvelles approches théoriques, dont la théorie des parties prenantes (Freeman, 2002; Freeman et al, 2010, Hasnas, 2012). "Certaines de ces approches mettent l'accent sur la sauvegarde et l'équilibre des intérêts contradictoires des parties prenantes dans un mode de management qui maintient tout le monde dans une coalition pour la création de la valeur » (Blair et Stout, 1999, p. 275, p. 303).

\subsubsection{Remise en question d'une vision pessimiste de la nature humaine}

Divers observateurs ont contesté les postulats et arguments fondamentaux sur lesquels s'appuie la théorie d'agence (Boncori et Mahieux, 2012). Cette dernière a été essentiellement développée à partir d'hypothèses négatives présumant l'homogénéité du comportement humain, montrant notamment les managers comme des êtres purement égoïstes et opportunistes à qui on ne peut faire confiance (Hirsch, Friedman et Koza, 1990). En concevant le problème de l'organisation sociale comme tributaire d'une supposée nature égocentrique de l'homme, et celui du manager comme consistant à faire du mal aux autres (actionnaires) en vue de satisfaire ses propres intérêts, les théoriciens de la théorie de l'agence se sont forgé la conviction qu'ils devaient trouver des mécanismes de gouvernance pour empêcher les dirigeants de «faire du mal », et les inciter à «faire le bien ». L'enjeu fondamental est d'arriver à trouver des mécanismes permettant à toutes les parties prenantes de participer à la production dans l'organisation et à la création de valeur pour l'ensemble de la société.

Cette surveillance des managers est perçue par ces derniers comme une menace à leur autonomie personnelle et diminue leur motivation intrinsèque. Elle endommage leur perception de soi et affecte leur démarche volontaire de coopération en vue de la performance (Ghoshal, 2005). Plusieurs chercheurs ont souligné les conséquences normatives 
importantes que peut avoir une théorie sur le comportement des acteurs sociaux et tout particulièrement des gestionnaires et des managers (Pesqueux, 2000). Une théorie n'est jamais neutre, voire amorale. Elle transmet inévitablement un contenu idéologique, c'est-à-dire qu'elle fait « la promotion d'un ensemble d'idées qui, fondé sur des hypothèses portant sur la nature humaine et le monde qui nous entoure, décrit et justifie un ordre social » (Boncori et Mahieux, 2012, p. 131-132). Ghoshal (2005) a illustré comment de mauvaises théories, comme la théorie de l'agence et ses postulats fondamentaux, " peuvent détruire de bonnes pratiques en management d'entreprise » $(2005$, p. 86 [notre traduction]). Il a particulièrement dénoncé l'impact de deux erreurs conceptuelles majeures, à savoir : la " prétention de connaissance » (le "pretense of knowledge » de Friedrich August von Hayek [1989]) et une « idéologie pessimiste » de la nature humaine (Ghoshal, 2005, p. 76 [notre traduction]). Le réductionnisme psychologisant de la théorie entendait justifier l'appropriation par les actionnaires de la valeur produite par l'entreprise.

Par effet de contamination, un jeu de domino, la vision pessimiste de la nature humaine et les présupposés de la théorie de l'agence qui en dérivent ont influencé les travaux d'institutions de recherche et de nombreux chercheurs en gestion; c'est notamment le cas de l'École de Chicago étudié par Ghoshal (2005). Cette vision a aussi modulé le développement des normes, des modes de gestion et d'organisation, bref tous les aspects de la gouvernance, ainsi que la production de techniques à la mode.

Il en découle ce que beaucoup perçoivent comme la prééminence d'une mentalité de lutte contre le feu et le recours prépondérant, souvent inconditionnel, à la théorie en management qui a pris les traits d'engouements et de modes managériaux comme les cercles de qualité, le JAT (juste à temps), le BPR (Business Process Reengineering), la méthode Six Sigma, etc., avec des managers qui attendent du recours, même isolé, à ces outils ou procédés qu'il les aide à aborder leurs problèmes plus larges et à produire des richesses (Davies, 2012, p. 98).

Toutefois, les dirigeants peuvent mal poser leurs problèmes conduisant à des résultats voulus à court terme, mais qui font empirer les problèmes à long terme. L'attribution de stock-options comme mécanisme incitatif des dirigeants pousse en effet ces derniers à gonfler les résultats comptables à court terme en vue de maximiser leurs rémunérations. Ce mécanisme incitatif (stock-options) a permis un enrichissement spectaculaire des dirigeants et a conduit à des scandales financiers très connus comme Enron et Worldcom.

\subsubsection{Les actionnaires ne sont pas les seuls « investisseurs » dans l'entreprise}

À l'instar des juristes, les critiques des économistes et des financiers ont fait valoir que le statut de l'actionnaire n'est pas celui d'un propriétaire de l'entreprise; il possède simplement un droit résiduel aux flux monétaires produits par l'entreprise. Ces critiques ont rappelé que la valeur d'une entreprise est créée non seulement avec l'apport financier des actionnaires, mais grâce à une combinaison de ressources apportées par différentes parties prenantes. «Les employés et les gestionnaires apportent leur capital humain, tandis que les actionnaires contribuent au capital financier »(Ghoshal, 2005, p. 80 [notre traduction]). Le terme "investisseur » doit être élargi pour aller au-delà d'une logique purement financière (Lordon, 2002). Toutes les parties prenantes s'investissent dans une organisation ou une entreprise. Les employés investissent du temps, des efforts; l'État forme des employés, les soigne, construit les infrastructures; les communautés produisent des travailleurs motivés, investissent en ressources collectives, en infrastructures et consomment les productions de l'entreprise. Les autres entreprises constituent un environnement sans lequel l'activité économique demeure sans objet. Comme la création de valeur est obtenue en combinant toutes ces ressources, les motifs favorisant la distribution de la valeur aux seuls actionnaires de l'entreprise paraissent dénués de fondement.

Lan et Heracleous (2010) suggèrent, pour leur part, de réexaminer de façon critique trois dimensions caractérisant l'entreprise. Ainsi, ils considèrent que dans la relation "principal-agent", les actionnaires détiennent plutôt des parts de l'entreprise. De plus, dans la relation "principal-agent ", les dirigeants ne sont pas des subordonnés des actionnaires; ils sont 
plutôt des fiduciaires autonomes, investis du pouvoir d'agir au nom et au bénéfice des parties prenantes. Enfin, le conseil d'administration n'a pas pour rôle principal de surveiller les gestionnaires pour que leurs intérêts ne divergent pas de ceux des actionnaires, mais exerce plutôt un rôle de médiateur hiérarque, un organe qui veille à équilibrer les revendications et les intérêts souvent contradictoires des parties prenantes. Ainsi, Lan et Heracleous (2010) remettent en question la personnification de l'entreprise et l'objectif ultime de la maximisation de la richesse des actionnaires. Ils proposent une nouvelle conception de l'entreprise reposant sur un principe de "team production" qui met de l'avant la primauté du conseil d'administration.
En outre, plutôt que la réduction des conflits d'agence entre les dirigeants (agents) et les actionnaires (principal) telle que préconisée par la théorie d'agence, la conception de «team production » favorise l'alignement des intérêts entre la direction (membre de l'équipe) et l'entreprise (principal) par le conseil d'administration qui a alors pour rôle de limiter les conflits d'intérêts de l'ensemble de l'équipe de production (Lan et Heracleous, 2010). Ainsi, le principal enjeu économique de cette conception ne réside pas dans la façon de minimiser les coûts d'agence, mais se trouve plutôt dans la façon de maximiser le potentiel d'investissements spécifiques de toutes les parties prenantes leur permettant de participer à l'équipe de production et à la création de valeur pour l'entreprise.

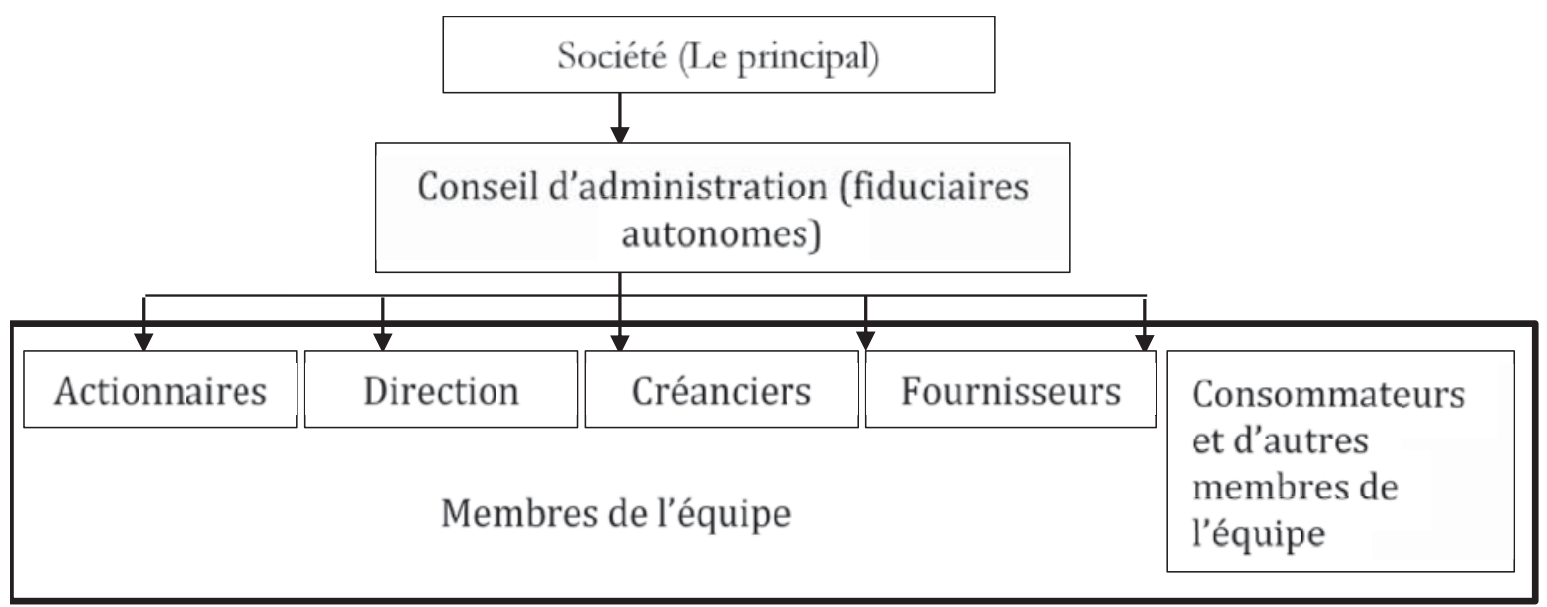

Figure 2 - Le modèle de la primauté du conseil d'administration Source : Modèle de Lan et Heracleous, L. (2010), p. 302. (Notre traduction)

Contrairement à la perspective actionnariale dans laquelle s'inscrit la théorie de l'agence et qui réduit les objectifs de la gouvernance d'entreprise à la valeur actionnariale, les théories récentes de l'entreprise issues de nombreux travaux de recherche montrent le caractère nécessaire de la prise en compte des intérêts des différentes parties pour la création de la valeur (Segrestin et Hatchuel, 2011, p. 10). Elles préconisent ainsi une gouvernance pluraliste susceptible de créer un avantage concurrentiel des entreprises (Bosse et al., 2009; Harrison et al., 2010 dans Segrestin et Hatchuel, 2011, p. 10). Segrestin, et Hatchuel (2011) se demandent si le cadre juridique actuel de gouvernance d'entreprise permet de concilier les intérêts de toutes les parties prenantes de manière neutre dans une perspective partenariale et de limiter les risques d'opportunisme des dirigeants. Selon Segrestin et Hatchuel (2011), bien que de grands pouvoirs soient donnés aux dirigeants d'entreprise, le droit n'encadre pas suffisamment les conditions d'exercice de ces pouvoirs, ce qui pourrait en partie expliquer la persistance de la doctrine actionnariale standard au détriment de la perspective partenariale.

Pour défendre cette hypothèse et illustrer la nécessité de revisiter le droit des sociétés pour instaurer une forme de gouvernance partenariale, Segrestin et Hatchuel (2011) s'appuient sur la métaphore du pouvoir de gestion du capitaine de navire encadré par la règle dite « des avaries communes ». En effet, ce dernier peut jeter, en cas de tempête, de la marchandise pour faire du lest (Segrestin et Hatchuel, 
2011, p. 21). Cette règle dite « des avaries communes » stipule que des marchandises à bord pourront être sacrifiées dans l'intérêt commun du navire et de la cargaison. Cette règle peut être appliquée dans l'entreprise pour sacrifier quelques intérêts particuliers (actionnaires) au nom d'un intérêt commun (fournisseurs, clients, banquiers ou prestataires de services). L'efficacité de la règle des «avaries communes » amène Segrestin et Hatchuel (2011) à considérer le cadre juridique comme un modèle destiné à empêcher certaines parties prenantes de l'entreprise, notamment les actionnaires, d'influencer les décisions de gestion des dirigeants, et permettant également de mutualiser davantage les dommages liés à une décision de gestion nécessaire à l'intérêt commun.

\subsubsection{L'impact de la financiarisation de l'économie}

Un chercheur critique, Gilles Bourque, examinant l'impact de la financiarisation sur l'économie réelle au Canada, démontre que le modèle de gouvernance axé sur la maximisation de l'avoir des actionnaires a fortement appauvri la société en général, et particulièrement les travailleurs salariés. Le transfert de la valeur aux actionnaires (dividendes versés) a connu depuis 1995 au Canada une croissance spectaculaire. En effet, la rentabilité de l'investissement a reçu un traitement privilégié au détriment d'une croissance des entreprises qui assure à ces dernières leur pérennité. Cette situation conduit à un surcoût de capital, à un prélèvement financier accru sur la richesse produite sans création de nouvelles valeurs ajoutées (Bourque, 2014). Ce surcoût du capital, dans les conditions décrites précédemment, conduit à la diminution des revenus perçus par les autres parties prenantes de la firme et en premier lieu par les salariés. La firme n'ayant plus d'objectifs sociaux, seuls les individus qui la constituent ont des objectifs et ces individus en viennent à contracter librement entre eux de façon à maximiser leur avoir financier, peu n'importe les coûts sociaux, individuels et le sort de l'entreprise. D'autres chercheurs sont venus renchérir dans cette critique :

Que la rentabilité financière soit une préoccupation des dirigeants, de l'encadrement et de l'ensemble des acteurs au sein des organisations, chacun peut le comprendre et l'admettre. Un vrai problème se pose toutefois à partir du moment où, au nom de cette nécessité, on assiste à une financiarisation de la gestion. Celle-ci conduit à traduire et évaluer l'ensemble des activités à l'aune d'indicateurs et de ratios financiers. Lorsque la logique financière s'impose comme logique dominante et quasi exclusive, elle met en péril la société elle-même, ses normes, ses valeurs, ses finalités (Gaulejac et Hanique, 2015, p. 67).

Dans un contexte de généralisation de cette pratique de surcoût de capital due à la globalisation et à la mondialisation, la société cotée en Bourse peut être vendue au plus offrant (un individu ou un groupe devenant majoritaire dans le capital), même s'il s'agit d'un « raider» désireux de la démanteler, de délocaliser ses opérations vers des zones moins coûteuses en matière de salariat et de coûts totaux de production, ou moins surveillées sur le plan environnemental. L'économie réelle qui devrait être gouvernée dans une perspective à long terme se voit imposer des diktats et des rendements excessifs par des actionnaires ou des marchés financiers, la règle financière à court terme supplantant les stratégies de développement à long terme (Robé, 2009).

Cette dominance des actionnaires ou des marchés financiers sur l'économie mondiale s'est fait tristement remarquer avec la crise déclenchée en 20072008, crise qui n'est pas que financière. «La finance est devenue un monde qui permet l'enrichissement d'individus sans qu'il y ait nécessairement création de richesse » (Bourque, 2014, p. 29). Le problème fondamental du renouvellement du capital n'a pas pour autant été réglé par ces pratiques financières, car le taux net d'accumulation du capital est toujours en baisse. Selon Bourque, « le point de vue actionnarial, qui domine aujourd'hui l'entreprise, privilégie en effet la rentabilité de l'investissement au lieu de la croissance et favorise la distribution massive des profits aux actionnaires »(Bourque, 2014, p. 37).

[...] le credo de création de valeur actionnariale se double d'une autre obsession normative : la distribution du « cash-flow libre » aux investisseurs. Il s'agit d'éviter que les dirigeants ne le retiennent en raison de leurs propres intérêts et/ou pour réaliser des investissements non rentables. Le mot d'ordre assigné à la fonction managériale est donc d'extraire (disgorge/ discharge) le cash-flow libre et de le 
rendre aux actionnaires sous la forme principale des dividendes, de procéder à des rachats d'actions (share repurchase ou stock buyback), ou encore de le rendre aux prêteurs (Boncori et Mahieux, 2012, p. 134).

En effet, dans l'environnement mondialisé actuel, les organisations deviennent de plus en plus importantes et multinationales; le chiffre d'affaires de certaines organisations dépasse le produit intérieur brut de nombreux États (Rousseau et Tchotourian, 2012). On a pu observer que « la gouvernance des sociétés par actions constitue un élément déterminant de la croissance, du développement et du succès des sociétés dans le contexte de la mondialisation des marchés » (Rousseau et Tchotourian, 2012, p. 15).

L'«idéologie libérale », ayant pour objectif principal d'exclure du domaine de la science du management les préoccupations d'ordre éthique, a été largement diffusée (Ghoshal, 2005). Et cette diffusion s'est étendue à des disciplines telles que le droit, la sociologie, la psychologie sociale et la plupart des autres disciplines de base (Ghoshal, 2005). Comment renverser cette tendance? Comme le souligne Ghoshal (2005), si la tendance actuelle dans les théories de gestion des entreprises doit être inversée, cela doit être l'œuvre des universitaires des écoles de management puisque, à travers elles, sont diffusées toutes les théories qui influencent et modifient les comportements des managers et des futurs managers.

\subsection{La perspective sociologique et le caractère institutionnel des organisations}

Un regard critique montre que la théorie de l'agence n'est pas une " théorie » au sens d'une production scientifique éprouvée, mais plutôt une doctrine qui ne peut expliquer la réalité de l'entreprise et du monde où elle intervient (Krugman, 2009). C'est pourquoi de nombreux sociologues et juristes ont voulu démontrer que l'analyse institutionnelle rend bien mieux compte de la nature des organisations et de leurs relations avec le cadre social ambiant. Bien que la Cour suprême du Canada ait fait évoluer le débat dans des jugements de 2004 et 2008 en élargissant considérablement les principes de gouvernance des sociétés par actions (malgré la place importante qu'elle accorde toujours au business judgment rule), elle n'est pas allée jusqu'à reconnaitre cette nature institutionnelle, c'est- à-dire à la percevoir comme un phénomène de volonté collective. Dans l'approche institutionnelle, la société par actions et les entreprises qu'elle exploite se concrétisent et s'inscrivent dans la durée comme un projet social beaucoup plus large. L'impact de ses décisions de toutes natures ne permet plus de limiter ses devoirs aux obligations de droit privé reconnus pour une personne morale, soit le même statut accordé aux personnes physiques. Dans cette perspective, dès les années 1930, Berle et Means proposaient une vision beaucoup plus adéquate de la réalité économique et gouvernementale de la société par actions et du rôle de ses dirigeants et administrateurs :

The economic power in the hands of the few persons who control a giant corporation is a tremendous force which can harm or benefit a multitude of individuals, affect whole districts, shift the currents of trade, bring ruin to one community and prosperity to another. The organizations which they control have passed far beyond the realm of private enterprise - they have become more nearly social institutions (Berle et Means, 1932, p. 46).

On a eu tendance depuis à oublier que l'entreprise n'est jamais seule au monde, et qu'elle ne crée pas le gros des formes qui la concrétisent. Elle surgit et existe grâce aux institutions qui l'ont précédé, de l'État qui la met au monde par des statuts de constitution, à la société ambiante qui lui procure sa raison d'être, ses ressources, ses traditions et outumes. C'est dans cet ensemble particularisé par une culture donnée qu'elle adopte un ensemble de pratiques orientées à des fins. Et ces fins ne se limitent jamais à ses seuls objectifs particuliers d'entreprise. Selon Jean-Guy Belley et François Dupuis, la société par actions a tiré profit, dans un contexte donné, d'un statut juridique plus proche de celui de l'État que celui des individus :

Rangée dans la catégorie des gouvernements, la société par actions rejoint le groupe des entités habilitées à créer du droit objectif, sous forme de règles d'application générale ou de décisions individualisées opposables à autrui. La théorie qui convient à l'analyse des décisions de ses dirigeants est dans ce cas la théorie de l'agent juridique plutôt que celle du mandataire représentant un sujet de droit dans 
l'exercice de ses droits subjectifs. Contrairement à ce dernier, l'agent juridique exerce un pouvoir institutionnel. Ce pouvoir s'analyse comme une aptitude à prendre des décisions de type fondamentalement politique en ce qu'elles peuvent légitimement affecter les intérêts d'autrui $[\ldots]$ même à l'encontre de sa volonté ou sans son consentement préalable (Belley et Dupuis, 2013, p. 217).

Par ailleurs, il convient de ne pas négliger ce que les sciences humaines ont réalisé depuis une cinquantaine d'années. Au cadre d'analyse sociologique traditionnel, portant sur des sociétés globales, s'est jointe l'analyse d'une mondialisation des rapports sociaux, de l'impérialisme de certaines nations et de leur lutte acharnée pour la gouverne du monde. Dans ce cadre d'analyse, on constate que l'action d'entreprendre prend place dans un tout complexe à dominante d'envergure mondial et qu'elle peut se lier à d'autres firmes pour constituer des oligopoles extrêmement puissants et potentiellement destructeurs (Morin, 2015). C'est ce que montrent les études portant sur l'oligopole bancaire anglo-américain et celui des grandes firmes transnationales, leur usage commun de la norme comptable «marked to market » découlant de la théorie de l'agence. La dilution des responsabilités dans cet univers s'avère catastrophique. Comme Belley, Robé assimile la prise de décision de ces firmes à une capacité de nature gouvernementale qui vient même surdéterminer la prise de décision propre aux États nationaux, aux instances internationales, au point de reléguer au second plan les nations elles-mêmes. «Given the shortcomings of the apparatus of norm creation at the international level, large firms must be understood as part of the political system of allocation of resources » (Robé, 2011, p. 79).

Si la « théorie » de l'agence, principalement axée sur la gouvernance interne et la disposition du produit financier, ne pouvait être vérifiée ou falsifiée, selon le principe de Popper, le fait qu'elle se soit propagée n'a pas manqué de créer ces indéniables « effets de réalité » que nous venons d'évoquer pour ce qui est de la conduite et de la perception des organisations. Ce système de pensée, une fois partagé, prend le caractère d'une prophétie autoréalisatrice (Merton, 1965) qui a pour effet de modifier les comportements. On a pu constater le déplacement des devoirs de loyauté, de prudence et de diligence au sein des conseils d'administration. On observe dans ce déplacement de responsabilité un effet de l'autorité de ceux qui énoncent la prophétie, par focalisation des esprits sur le « réel » ainsi affirmé. Ce processus insidieux a pour résultat de créer une réalité inadéquate et déformée, une réalité sur base d'illusions, aux effets pourtant bien réels. Ce champ de conscience, l'immédiat des acteurs, essentiellement non critique, finit par occuper la place de la théorie, à s'autoreproduire sous couverture de scientificité, ce que le pourtant très "libéral » Friedrich von Hayek (1989) appelait « the pretense of knowledge ». La conscience immédiate, non critique, repousse l'éthique sous le tapis de l'inconscient, à l'enseigne de l'évidence, avec pour conséquences, ces dérives, ces crises qui se reproduisent avec une extrême régularité. La corruption de la pensée se manifeste dans la corruption des comportements, des institutions économiques et politiques (Denault, 2017; OCDE, 2014).

\subsection{Le droit canadien et la théorie de l'agence}

Au Canada, les assises juridiques de la théorie de l'agence sont pratiquement inexistantes. Aucun texte de loi ou réglementaire canadien régissant les sociétés par actions, comme la Loi canadienne sur les sociétés par actions, L.R.C. 1985, c. C-44 (LCSA) 8 ou encore la Loi sur les sociétés par actions du Québec, L.R.Q., c. S-31.1 (LSAQ), ne reconnaît que les actionnaires par leur investissement financier se trouvent « propriétaires » d'une partie ou d'une proportion des actifs (ou de l'entreprise) et encore moins de la société par actions qui lui sert de support juridique. En effet, la société par actions est une entité morale, distincte de ses membres (actionnaires, administrateurs, dirigeants, etc.), qui existe par elle-même. Propriétaire de ses propres biens et capitaux, elle peut acheter des actifs et passer les contrats nécessaires à ses activités (Robé, 2009)9. Selon Paul Martel, ce principe s'est imposé depuis de nombreuses années :

La jurisprudence a établi qu'une action n'est pas un titre de propriété sur les biens de la société $[\ldots]$, pas plus qu'un titre de créance. En effet, l'actionnaire n'est pas un créancier de la société; au contraire, la loi établit divers mécanismes visant à s'assurer que les actionnaires passent toujours après les créanciers lors de toute sortie de fonds ou de répartition de biens de la société (Martel, 2014, p. 88). 
En apportant les capitaux en risque dont la société par actions a besoin pour exploiter sa ou ses entreprises, les actionnaires se voient, par les statuts de constitution, conférer des droits et des privilèges, tels le droit de participer aux assemblées d'actionnaires, le droit de recevoir les dividendes déclarés, le droit de se partager le reliquat des biens de la société lors de sa liquidation, de nommer ou de révoquer les membres du conseil d'administration. Advenant des difficultés financières de la société par actions, la responsabilité des actionnaires est limitée; ils ne perdent que leur mise de fonds ${ }^{10}$. Si les actifs de la société par actions sont insuffisants pour payer tous ses créanciers, ces derniers ne pourront pas combler le déficit en saisissant et en vendant les biens personnels des actionnaires ou des administrateurs.

Et de fait, aucun texte légal canadien ne prescrit une obligation au conseil d'administration et aux dirigeants d'une société par actions d'agir comme les " fiduciaires ", comme les agents des actionnaires ou encore de maximiser la valeur actionnariale (shareholder value) représentée par la théorie de l'agence « comme l'incarnation de l'intérêt général, conforme au bien commun » (Gaulejac et Hanique, 2015, p. 65). Parmi les textes majeurs pour nous l'enseigner, la LCSA, une pièce majeure du droit des sociétés au Canada depuis 1985, établit que les administrateurs et les dirigeants doivent, dans l'exercice de leurs fonctions, agir en premier lieu avec intégrité et de bonne foi "au mieux des intérêts de la société »; et en second lieu, avec le soin, la diligence et la compétence dont ferait preuve, en pareilles circonstances, une personne prudente (art. 122). Par ailleurs, le Code civil du Québec rejoint les objectifs de la LCSA depuis 1994 avec les articles suivants :

321. L'administrateur est considéré comme mandataire de la personne morale. Il doit, dans l'exercice de ses fonctions, respecter les obligations que la loi, l'acte constitutif et les règlements lui imposent et agir dans les limites des pouvoirs qui lui sont conférés.

322. L'administrateur doit agir avec prudence et diligence.

Il y est aussi stipulé que l'administrateur doit agir avec honnêteté et loyauté dans l'intérêt de la personne morale. Pourtant, en dépit de ces textes législatifs clairs, les tribunaux canadiens ont privilégié durant des décennies une conception très financière de l'intérêt de l'entreprise, sous l'emprise des préceptes de la «corporate governance » et de la théorie de l'agence. Selon plusieurs observateurs, cette interprétation dérive en droite ligne d'une décision rendue en 1919 par la Cour suprême du Michigan (États-Unis) soit l'affaire Dodge v. Ford Motor Co., 170 N.W. 668 (Tchotourian, 2014). Henry Ford souhaitait diminuer le prix de vente des véhicules qu'il produisait, les rendre accessibles au plus grand nombre, et engager de nouveaux employés, plutôt que de redistribuer les profits aux actionnaires. Il désirait prendre en compte d'autres parties prenantes, comme les consommateurs, les employés, l'envergure à long terme de la société, etc. Cette décision d'affaires de Ford fut invalidée par la Cour, érigeant de ce fait les actionnaires comme les principaux ayants droit de la compagnie :

A business corporation is organized and carried on primarily for the profit of the stockholders. The powers of the directors are to be employed for that end. The discretion of directors is to be exercised in the choice of means to attain that end, and does not extend to a change in the end itself, to the reduction of profits, or to the non distribution of profits among its stockholders in order to devote them to other purposes (Dodge, p. 683).

Au Canada, deux jugements récents de la Cour suprême sont venus ébranler ce dogme centenaire de la primauté des intérêts des actionnaires ${ }^{11}$. Dans l'affaire des magasins Peoples, la cour parut très audacieuse en 2004 , lorsqu'elle se montra favorable à une conception plus large de l'« intérêt social » de la société par actions (Tchotourian, 2014). Le nœud de cette affaire, c'est que Wise acquiert en 1992 les magasins Peoples en recourant à un financement très complexe. Mais devant la concurrence de Walmart, en 1994, Wise et Peoples font faillite. Plusieurs fournisseurs ne sont pas remboursés. Se sentant floués, ils recourent aux tribunaux. La Cour suprême réfute l'interprétation voulant que l'intérêt de la société (l'entreprise) doive être assimilé à l'intérêt seul des actionnaires :

Pour ce qui est de l'obligation fiduciaire prévue par la loi, il est évident qu'il ne faut pas interpréter l'expression « au mieux des intérêts de la société » comme si elle signifiait simple- 
ment « au mieux des intérêts des actionnaires ». D’un point de vue économique, l'expression « au mieux des intérêts de la société »s'entend de la maximisation de la valeur de l'entreprise (par. 42).

Dans son jugement, la Cour suprême présente les facteurs à prendre en considération par les membres d'un conseil d'administration dans la gouvernance d'une entreprise pour atteindre cet objectif :

Nous considérons qu'il est juste d'affirmer en droit que, pour déterminer s'il agit au mieux des intérêts de la société, il peut être légitime pour le conseil d'administration, vu l'ensemble des circonstances dans un cas donné, de tenir compte notamment des intérêts des actionnaires, des employés, des fournisseurs, des créanciers, des consommateurs, des gouvernements et de l'environnement (par. 42).

En 2008, un deuxième signal dans ce sens est émis par la Cour suprême du Canada, dans l'affaire BCE. Il s'agit de la vente de BCE inc. à un consortium d'investissement privé à la tête duquel se trouvait le Conseil du régime de retraite des enseignants et enseignantes de l'Ontario (Teachers) (Chapman et al, 2008). Cette affaire portait sur l'approbation, par les tribunaux, d'un projet d'acquisition de BCE inc., au moyen d'un emprunt de 52 milliards de dollars. Certains détenteurs de débentures remettent en question l'opération, prétendant qu'elle est abusive et qu'elle ne respecte pas le critère de « caractère équitable et raisonnable » requis pour obtenir l'approbation par le tribunal d'un plan d'arrangement en vertu de la LCSA. Avec la transaction, la valeur boursière des débentures s'en trouverait modifiée. La décision de la Cour suprême précise que lorsque les administrateurs regardent les différents intérêts qu'ils doivent prendre en compte pour déterminer ce qui est le mieux pour l'« intérêt de la société », ils doivent agir de façon équitable et responsable.

Dans son ensemble, la jurisprudence en matière d'abus confirme que l'obligation des administrateurs d'agir au mieux des intérêts de la société inclut le devoir de traiter de façon juste et équitable chaque partie intéressée touchée par les actes de la société. Il n'existe pas de règles absolues. Il faut se demander chaque fois si, dans les circonstances, les administrateurs ont agi au mieux des intérêts de la société, en prenant en considération tous les facteurs pertinents, ce qui inclut, sans s'y limiter, la nécessité de traiter les parties intéressées qui sont touchées de façon équitable, conformément aux obligations de la société en tant qu'entreprise socialement responsable (par. 82).

Ces deux jugements impliquent qu'en vertu de leur devoir de loyauté, de prudence et diligence, les administrateurs et les dirigeants doivent veiller aux intérêts propres de la société qui peuvent être différents de ceux des actionnaires. La gouvernance ne peut pas se restreindre à un horizon temporel de profit à court terme. La Cour suprême marque aussi ses distances à l'endroit d'une gouvernance privilégiant certains principes de la théorie de l'agence. Selon plusieurs observateurs,

[...] la Cour propose un nouveau regard sur l'entreprise : il s'agit d'une entité autonome avec des objectifs légitimes construits en relation avec une pluralité de parties prenantes qu'elle doit considérer de manière juste et équitable (Gendron, 2013, p. 142; Rousseau et Tchotourian, 2012).

Confirmant la doctrine émanant de ces jugements importants de la Cour suprême du Canada, les législateurs canadien et québécois sont intervenus depuis quelques années sur les plans législatif et réglementaire pour responsabiliser l'entreprise et établir des balises à la gouvernance des sociétés qui dépassent les postulats de la théorie de l'agence et de sa vision strictement financière ou économique de l'entreprise. En effet, on retrouve de plus en plus de lois imposant aux administrateurs et aux dirigeants des sociétés par actions des obligations qui engagent leur responsabilité personnelle civile, voire pénale, vis-à-vis de nombreuses parties prenantes (employés, créanciers, gouvernements, populations, etc.). Le professeur Rousseau (2012) dresse une synthèse de quelque 260 dispositions législatives s'appliquant aux administrateurs et dirigeants de sociétés faisant affaire au Québec dans des domaines d'application aussi variés que l'agriculture, l'environnement, le patrimoine, la consommation, l'énergie, la faillite, la fiscalité, l'importation, le transport, le travail, la santé et la sécurité, les valeurs mobilières, les services financiers, etc. Bref, autant de critères venant encadrer la gouvernance des sociétés.

Ainsi, l'adoption en 2006 de la Loi québécoise sur le développement durable (L.R.Q., c. D-8.1.1), par l'Assemblée nationale, vient-elle changer la donne, 
même si les entreprises privées ne sont qu'indirectement assujetties à cette loi. Elle impose en effet à l'administration publique québécoise un cadre de gestion qui s'inscrit dans la recherche d'« un développement qui répond aux besoins du présent sans compromettre la capacité des générations futures à répondre aux leurs »; les entreprises privées devront cependant y souscrire si elles veulent participer aux projets de l'État québécois. En effet, depuis 2008, la Loi sur les contrats des organismes publics (L.R.Q., c. C-65.1) et ses règlements d'application ${ }^{12}$ permettent à l'administration publique québécoise de considérer l'apport d'un système d'assurance de la qualité, notamment une norme ISO, ou une spécification liée au développement durable, à la protection des parties prenantes et à l'environnement, pour la réalisation d'un contrat. L'organisme public peut permettre à tout fournisseur, à tout prestataire de services ou à tout entrepreneur, de présenter une soumission ainsi qualifiée et accorder à celui qui répond à l'exigence, une marge préférentielle pouvant atteindre $10 \%$.

Divers jugements rendus par des tribunaux canadiens sont aussi venus ouvrir le jeu en matière de responsabilité sociale des entreprises. À titre d'exemple, dans l'affaire Ciment du Saint-Laurent ${ }^{13}$, la Cour suprême du Canada a sanctionné une responsabilité sans faute dans une situation où des voisins subissaient des inconvénients (troubles) anormaux ou excessifs. Un recours collectif est engagé, en 1994, par les résidents voisins d'une cimenterie de Beauport, près de Québec, pour les préjudices subis en raison des poussières, du bruit et des odeurs de la cimenterie. La cour confirme la condamnation de Ciment du Saint-Laurent l'obligeant à des dommages-intérêts même si elle n'avait en principe commis aucune faute relativement aux normes environnementales légales applicables. En effet, l'entreprise respectait les normes environnementales applicables et avait mis en place les meilleurs équipements antipollution connus. Bref, en ajoutant la responsabilité objective (imputation des risques) à la responsabilité subjective (imputation d'une faute), la Cour suprême du Canada est venue en quelque sorte faire monter davantage la pression en faveur de la prudence, de la prévention, du dialogue éthique et de la concertation entre voisins, entre partenaires ou parties prenantes de la vie sociale.
Depuis quelques années, législateurs et tribunaux du Canada ne s'en tiennent plus à une vision strictement économique de l'entreprise; ils élargissent considérablement le devoir de loyauté des dirigeants et des administrateurs de sociétés par actions aux autres parties prenantes. Mais il reste un long chemin à parcourir pour que les postulats de la théorie de l'agence « comme conception dominante de la gouvernance d'entreprise » soient complètement rejetés. À titre d'exemple, la Cour suprême du Canada dans l'affaire Peoples, de façon un peu contradictoire, n'hésite pas à appliquer à la LCSA la règle de l'appréciation commerciale (Business Judgment $\mathrm{Rule}$ ) issue de la common law. Cette règle de retenue judiciaire a traditionnellement eu pour objectif de "protéger l'indépendance du conseil d'administration dans la gestion des affaires de la société » (Tchotourian, 2014, p. 95). Les tribunaux ont depuis longtemps préféré s’ingérer le moins possible dans les litiges portant sur des décisions d'affaires des administrateurs. Pour la Cour suprême, cette règle d'interprétation demeure tant que les décisions prises par les conseils d'administration constituent des décisions raisonnables au vu de ce qu'ils savent ou devraient savoir.

Lorsqu'il s'agit de déterminer si les administrateurs ont manqué à leur obligation de diligence, il convient de répéter que l'on n'exige pas d'eux la perfection. Les tribunaux ne doivent pas substituer leur opinion à celle des administrateurs qui ont utilisé leur expertise commerciale pour évaluer les considérations qui entrent dans la prise de décision des sociétés. Ils sont toutefois en mesure d'établir, à partir des faits de chaque cas, si l'on a exercé le degré de prudence et de diligence nécessaire pour en arriver à ce qu'on prétend être une décision d'affaires raisonnable au moment où elle a été prise (par. 67).

Le professeur Tchotourian soulève un problème réel lorsqu'il affirme qu'une telle retenue des juges « constitue un obstacle potentiel au mouvement de responsabilisation sociétale des administrateurs de sociétés » (2014, p. 102). L'application de la règle du Business Judgment Rule peut en effet protéger de facto l'esprit de la théorie de l'agence en conférant aux dirigeants et aux administrateurs le statut de fiduciaire des actionnaires. Un contrôle judiciaire moins sévère risque alors de soutenir une délibération éthique moins exi- 
geante de la part des administrateurs. En cas d'arbitrage entre les considérations financières, sociales et environnementales, le critère financier risque de s'imposer plus facilement dans l'attente d'une approche plus laxiste des tribunaux.

Plus encore, la plupart des recours visant à protéger les parties prenantes d'une décision abusive, inéquitable, ou d'une oppression des administrateurs et dirigeants d'une société par actions ne sont souvent disponibles qu'à un nombre limité d'entre elles ${ }^{14}$. Dans la pratique, seuls les actionnaires, les détenteurs de débentures et les créanciers sont autorisés à accéder aux tribunaux (Allaire et Rousseau, 2014; VanDuzer, 2010; Martin, 2012). Un tel accès privilégié des actionnaires et des créanciers peut justifier que les administrateurs et les dirigeants d'une société par actions placent leurs intérêts en premier afin d'éviter les litiges. Cela peut secondariser la prise en compte effective des intérêts des autres parties prenantes, comme les travailleurs et les communautés (Martin, 2014).

Finalement, il importe de garder à l'esprit que la portée et l'efficacité des législations nationales (comme celles du Canada et du Québec) peuvent être assez limitées. Avec la globalisation de l'économie, « le champ d'action des entreprises n'est pas limité par le territoire de tel ou tel État » (Robé, 2009, p. 35). Les États sont désormais mis en concurrence sur les plans législatif, normatif et fiscal. Les multinationales ou les sociétés transnationales passent donc de plus en plus facilement entre les mailles des filets prudentiels. Leur expertise financière et légale permet de créer des entités économiques en réseaux complexes (filiales, agences, etc.) opérant partout dans le monde afin, justement, d'échapper aux contraintes émanant des États nationaux. Si leurs comportements ont des conséquences économiques graves sur les citoyens (travailleurs, consommateurs, etc.), les États ne peuvent ou ne veulent pas toujours intervenir afin de sanctionner les situations, alors même qu' " un droit postmoderne émerge » sur le plan international pour appréhender la nature des entreprises dans leur diversité (Tchotourian, 2014, p. 15; Benyekhlef, 2016).

\section{Conclusion}

En synthèse, peut-on encore se surprendre du caractère répétitif des désordres politiques, écono- miques et financiers actuels, et surtout de l'impossibilité de les résoudre par des interventions psychosociales visant à rectifier les conduites individuelles, ou même par quelques dispositifs institutionnels particuliers? La conscience du gestionnaire, du citoyen, fait face à une réalité d'un type différent, à des forces contradictoires qui nécessitent de savoir conjuguer économie, finance, droit, sociologie, sciences comptables, éthique et théorie du management, afin de sortir les acteurs sociaux d'un état d'incompréhension et de déresponsabilisation. La conscience spontanée a pour effet d'éradiquer l'univers des choix collectifs; sont privilégiés le caractère libertaire de l'action des acteurs économiques, la concurrence sous toutes ses formes sous la dictature du profit, et ce, peu importe les conséquences et les risques manifestes ou potentiels pour les collectivités. Ces valeurs ont reçu leur justification de modèles marqués par le «pretense of knowledge », dont une théorie de l'agence, qui a pour objectif explicite de propager une gouvernance d'entreprise dominée par la rhétorique financière. L'État, de régulateur qu'il était, est conduit à éponger les crises financières et économiques, à socialiser leurs coûts, à absorber les effets de l'« aléa moral ». Le régulateur, prêteur de dernier ressort, diffusant sur la collectivité le coût de ces désordres, est devenu le régulé, privé de revenus et de pouvoirs réglementaires. Cette dépendance étatique a pour effet d'accroître les dettes publiques et d'affaiblir toute gouvernance démocratique du développement. L'action de nombreux organismes internationaux a bel et bien propagé une idéologie avec les résultats désastreux que l'on constate aujourd'hui dans le monde (Krugman, 2009; Stiglitz, 2015; Ziegler, 2002).

Au plus fort de ces crises et événements financiers scandaleux, la solution courante consiste à cibler les individus, à imaginer un redressement éthique en plaçant l'accent sur les sphères d'action individuelles ou organisationnelles, sans toucher aux règles du jeu à l'échelon macrosocial, sans modifier les institutions dominantes qui influent inévitablement sur les comportements et les organisations. Les rapports sociaux étant complexes, leur gouvernance effective nécessite des approches interdisciplinaires renouvelées. Un mouvement de réforme requiert de concevoir la gouvernance des organisations et plus particulièrement des entreprises dans la perspective d'un développement institutionnel et universel où les collectivités, les institutions publiques, les professionnels, les individus, renouvellent leurs participations, en tant que cocréateurs, 
pour réaliser concrètement les «capabilités » d'une communauté humaine (Sen, 2010; Nussbaum, 2012). Dans une telle conjoncture, il est d'importance, à l'université, dans les formations professionnelles, de former à l'esprit critique, de comprendre les dimensions institutionnelles d'une problématique, les aspects variés de la responsabilité et les sources normatives en jeu. Il est alors nécessaire d'identifier les fausses théories, ce qui peut requérir de mobiliser des éléments de sociologie critique, une théorie des communs (Dardot et Laval, 2014), des pratiques ouvertes d'éthique appliquée comme l'entend Perrot (2011). Ce chantier critique multidisciplinaire fera apparaitre pour ce qu'elles sont les idéologies et les fausses théories, comme cette théorie de l'agence et valorisera d'autres approches jugées urgentes dans la réforme de la réalité sociale (Gond, 2013).

\section{NOTES}

1 Par ordre alphabétique.

2 « $[\ldots]$ there is one and only one social responsibility of business-to use its resources and engage in activities designed to increase its profits so long as it stays within the rules of the game, which is to say, engages in open and free competition without deception or fraud» (Friedman, 1970, p. 33).

3 La théorie d'agence s'est développée en deux branches : l'une positive, l'autre orientée principal-agent. La branche positive « se concentre non seulement sur l'identification des situations présentant des conflits d'intérêts entre principal et agent mais aussi sur la description des mécanismes de contrôle et coordination assurant l'efficacité organisationnelle ». La deuxième branche « rassemble des travaux à visée normative où est recherchée une structure de partage optimale du risque » (Boncori et Mahieux, 2012, p. 132-133).

4 À titre d'exemple, l'article 154 de la Loi sur les sociétés par actions, L.R.Q., c. S-31.1 mentionne : Les administrateurs de la société sont solidairement responsables envers ses employés, jusqu'à concurrence de six mois de salaire, pour les services rendus à la société pendant leur administration respective.

5 Art. 103 Loi sur les sociétés par actions, L.R.Q., c. S-31.1.

6 « Fiduciary obligations arise when one party to a contract has no choice but to repose trust in the other, and hence is vulnerable to the other's malfeasance or negligence » (Hasnas, 2012, p. 50).

7 L'organisation ou la firme dans le cadre de cette étude est une société anonyme par actions telle qu'on l'observe dans les pays de l'OCDE.

8 Repéré à https://www.ic.gc.ca/eic/site/cilp-pdci.nsf/fra/h_cl00867.html

9 Une personne morale a une existence perpétuelle.

10 Sauf si l'actionnaire a personnellement cautionné les dettes de la société par actions en faveur des créanciers.

11 Magasins Peoples (Syndic de) c. Wise [2004] 3 RCS 461; BCE c. Détenteurs de débentures 1976 [2008] 3 RCS 560.

12 Règlement sur les contrats d'approvisionnement des organismes publics, (R.R.Q., c. C-65.1, r.2); Règlement sur les contrats de services des organismes publics, (R.R.Q., c. C-65.1, r.4); Règlement sur les travaux de construction des organismes publics, (R.R.Q., c. C-65.1, r.5.

13 Ciment du Saint-Laurent inc. c. Barrette, [2008] 3 R.C.S. 392.

14 Art. 441 LSAQ; art. 241 LCSA.

\section{RÉFÉRENCES}

Alchian, A. et Demsetz, H. (1973). The property right paradigm. Journal of Economic History, 33, 16-27.

Allaire, Y. et Rousseau, S. (2014). Gouvernance et parties prenantes : l'obligation du conseil d'administration d'agir dans l'intérêt de la société. Institut de la gouvernance d'organisations privées et publiques IGOPP. Repéré à https://igopp.org/gouvernance-etparties-prenantes/.

Bardelli, P. (2012). La RSE au regard du rapport salarial. Dans C. Gendron, B. Girard (dir.), Repenser la responsabilité sociale de l'entreprise. L'école de Montréal (p. 77-90). Paris, France : Armand Colin/recherches.

Belley, J.-G. et Dupuis, F. (2013). La société par actions : « chose », « personne » ou « gouvernement » ? Dans P.-E. Moyse (dir.), Quelle performance? De l'efficacité sociale à l'entreprise citoyenne (p. 203-274). Montréal, Québec : Les Éditions Thémis. 
Benyekhlef, K. (2016). Vers un droit global? Montréal, Québec : Éditions Thémis.

Berle, A. et Means, G. (1932). The modern corporation and private property. New York, NY: The Macmillan Company.

Blair, M. et Stout, L. (1999). A team production theory of corporate law. Virginia Law Review, 85, 247-328.

Boncori, A. L. et Mahieux, X. (2012). Au-delà du bien et du mal. La théorie et les pratiques de gestion. Revue française de gestion, 38(228-229), 129-146.

Bosse et al. (2009). Stakeholders, reciprocity and firm performance. Strategic Management Journal, 30, 447-56.

Bourdieu, P. (1980). Le sens pratique, Paris, France : Éditions de Minuit.

Bourque, G. L. (2014). La financiarisation de l'économie nuit-elle aux entreprises ? L'Économie politique, 62(2), 29-38.

Boyer, R. (2015). Économie politique des capitalismes. Théorie de la régulation et des crises. Paris, France : La Découverte.

Boyer, R. (2011). Les financiers détruiront-ils le capitalisme? Paris, France : Economica.

Caballero, R. J. (2010). Macroeconomics after the crisis: Times to deal with the pretense-of- knowledge syndrome. The Journal of Economic Perspectives, 24(4), 85-102.

Chapman, R. D., DeLuca, F. A., Faley, J., Girvan, G. M., Hall, G. R., Hansen, R. O., Kerwin, E. P., Silver, B. (2008). L'acquisition par emprunt de BCE devant la Cour suprême du Canada : Précisions sur les obligations des administrateurs dans le cadre de prises de contrôle et lorsque les intérêts des parties intéressées sont en opposition. Repéré à : https://mccarthy.ca/fr/article_detail.aspx?id=4300.

Charreaux, G. et Wirtz, P. (2005). Gouvernance des entreprises : nouvelles perspectives. Paris, France : Economica.

Clegg, S. R. et Ross-Smith, A. (2003). Revising the boundaries: Management education and learning in a postpositivist world. Academy of Management Learning \& Education, 2(1), 85-98.

Dalton, D. R., Daily, C. M., Certo, S. T., et Roengpitya, R. (2003). Meta-analyses of financial performance and equity: Fusion or confusion? Academy of Management Journal, 46, 13-26.

Dalton, D. R., Hitt, M. A., Certo, S. T. et Dalton, C. M. (2007). The fundamental agency problem and its mitigation: Independence, equity and the market for corporate control. Academy of Management Annals, 1, 1-64.

Dardot, P. et Laval, C. (2014). Commun. Essai sur la révolution au XXIe siècle. Paris, France : La Découverte.

Davies, J. (2012). Théorie du management: une approche systémique pour comprendre les pratiques et les comportements des managers. Vie \& sciences de l'entreprise, (1), 95-95.

Denault, A. (2017). De quoi Total est-elle la somme? Multinationales et perversion du droit. Montréal, Québec : Éditons Écosociété.

Fay, P., Boisseau, L. (2014, 2 janvier). Wall Street domine une capitalisation mondiale qui a doublé en dix ans. Les Échos. Repéré à https://www.lesechos.fr/02/01/2014/LesEchos/21596-090-ECH_wall-street-domine-une-capitalisationmondiale-qui-a-double-en-dix-ans.htm

Freeman, R. E. (2002). Stakeholder theory of the modern corporation. Dans T. Donaldson, et al. (dir.), Ethical issues in business (7e éd., p. 44). Upper Saddle River, NJ : Prentice Hall.

Freeman, R. E. et al. (2010). Stakeholder theory: The state of the art. Cambridge, Royaume-Uni : Cambridge University Press.

Friedman, M. (1970). The social responsibility of business is to increase its profits. The New York Times Magazine, 13, 32-33.

Gaulejac, V. de et Hanique, F. (2015). Le capitalisme paradoxant. Un système qui rend fou. Paris, France : Seuil.

Gendron, C. (2013). La firme : portrait d'un objet ambigu. Dans C. Gendron, B. Girard (dir.), Repenser la responsabilité sociale de l'entreprise (p. 135-147). Montréal, Québec : Armand Colin.

Ghoshal, S. (2005). Bad management theories are destroying good management practices. Academy of Management Learning \& Education, 4(1) 75-91.

Ghoshal, S. et Moran, P. (1996). Bad for practice: A critique of the transaction cost theory. The Academy of Management Review, 21(1), 13-47. 
Gomez, P.-Y. (2009). La gouvernance actionnariale et financière : Une méprise théorique. Revue française de gestion, 35, $369-391$.

Gond, J.-P. (2013). La responsabilité sociale de l'entreprise comme praxis performative. Dans C. Gendron, B. Girard (dir.), Repenser la responsabilité sociale de l'entreprise. L'école de Montréal (p. 149-164). Montréal, Québec: Armand Colin.

Harrison et al. (2010). Managing for stakeholders, stakeholder utility functions and competitive advantage. Strategic Management Journal, 31, 58-74.

Hart, O. et Moore, J. (1990). Property rights and the nature of the firm. Journal of Political Economy, 98, 1119-1158.

Hasnas, J. (2012). Whither stakeholder theory? A guide for the perplexed revisited. Journal of Business Ethics, 112(1), 47-57.

Hirsch, P. M., Friedman, R. et Koza, M. P. (1990). Collaboration or paradigm shift? Caveat emptor and the risk of romance with economic models for strategy and policy research. Organization Science, 1, 87-98.

Krugman, P. (2009). What went wrong with economics. The Economist, 392 (8640).

Jensen M. et Meckling, W. (1994). The nature of man. The Journal of Applied Corporate Finance, 7(2), 4-19.

Jensen M. (2000). A theory of the firm : Governance, residual claims, and organizational forms. Cambridge, MA : Harvard University Press.

Jensen M. C. et Meckling, W. H. (1976). Theory of the firm : managerial behaviour, agency costs and ownership structure. Journal of Financial Economics, 3, 305-60.

Lan, L. L. et Heracleous, L. (2010). Rethinking agency theory: The view from law. Academy of Management Review, 35(2), 294-314.

La Porta et al. (1997). Legal determinants of external finance. Journal of Finance, 52(3), 1131-1150.

La Porta et al. (1997). Trust in large organizations, American Economic Review Paper and Proceedings, 87, 333-338.

La Porta et al. (1998). Law and finance. Journal of Political Economy, 106(6), 1113-1155.

La Porta et al. (1999). Corporate ownership around the world. The Journal of Finance, 54(2), 471-517.

Lordon, F. (2002). La politique du capital. Paris, France : Éditions Odile Jacob.

Maitland, I. (1994). The morality of the corporation: An empirical or normative disagreement? Business Ethics Quarterly, 4(4), 445-458.

Martel, P. (2014). La société par actions an Québec : les aspects juridiques. Montréal, Québec : Wilson et Lafleur.

Martin, I. (2014). Tying it all together: The potential of legal, social and market-based control mechanisms to enforce integrated and sustainable decision-making. Revue générale de droit, 44(2), 353-389.

Martin, I. (2012, 2 décembre). The impact of the regulatory environment on the definition of the best interests of the corporation. Repéré à https://ssrn.com/abstract $=2247434$.

Merton, R. K. (1965). Éléments de théorie et de méthode sociologique. Paris, France : Gérard Monfort.

Morin, F. (2015). L’hydre mondiale : L'oligopole bancaire. Montréal : Lux Éditeur.

Nussbaum, M. C. (2012). Capabilités : comment créer les conditions d'un monde plus juste? Paris, France : Climats.

OCDE (2014). The rationale for fighting corruption. CleanGovBiž Initiative. Integrity in practice. Repéré à https://www.oecd.org/cleangovbiz/49693613.pdf.

Orléan, A. (1999). Le Powvoir de la finance. Paris, France : Odile Jacob.

Perrot, É. (2011). Refus du risque et catastrophes financières. Paris, France_: Salavator. Repéré à https://fr.wikipedia.org/wiki/Paris.

Pesqueux, Y. (2000). Le gouvernement de l'entreprise comme idéologie. Paris, France : Ellipses.

Pfeffer, J. (2009). Renaissance and renewal in management studies: Relevance regained. European Management Review, 6, $141-148$.

Pfeffer, J. (2005). Why do bad management theory persist? A comment on Ghoshal. Academy of Management Learning \& Education, 4(1), 96-100. 
Robé, J.-P. (2009). À qui appartiennent les entreprises ? Le Débat, 3(155), 32-36.

Robé, J.-P. (2011). The legal structure of the firm. The Journal of Accounting, Economics \& Law: A Convivium, 1(1). Repéré à https://www.degruyter.com/downloadpdf/j/ael.2011.1.1/ael.2011.1.1.1001/ael.2011.1.1.1001.pdf.

Rouleau, L. (2007). Théories des organisations : approches classiques, contemporaines et de l'avant-garde. Québec, Québec : Presses de l’Université du Québec.

Rousseau, S. (2007). Théories contractuelles de la firme : Théorie du noud de contrats et théorie de l'agence. [Blogue Gouvernance. Idées et réflexions sur la gouvernance et les marchés financiers]. Repéré à http://droit-des-affaires.blogspot.ca/2007/02/thoriescontractuelles-de-la-firme_5992.html.

Rousseau, S. et Tchotourian, I. (2012). Pouvoirs, institution et gouvernance de la société par actions : lorsque le Canada remet en question le dogme de la primauté actionnariale. Les cahiers de la CRSDD, collection « Recherche », Montréal, Québec : UQAM, No 05-2012. Repéré à http://www.crsdd.uqam.ca/Pages/docs/05-2012.pdf.

Rousseau, S. (2012). La responsabilité civile et pénale des administrateurs : Tableau synoptique. Chaire en droit des affaires et gouvernance, 2012-03. Repéré à https://papyrus.bib.umontreal.ca/xmlui/handle/1866/6320.

Segrestin, B. et Hatchuel, A. (2011). Autorité de gestion et avaries communes : pour un complément du droit de l'entreprise? Finance Contrôle Stratégie, 14 (2), 9-36.

Sen, A. (2010). L'idée de justice. Paris, France : Flammarion.

Shleifer, A. et Wolfenzon, D. (2002). Investor protection and equity markets. Journal of Financial Economics, 66, 3-27.

Stiglitz, J. (2015). La grande facture. Les sociétés inégalitaires et ce que nous pouvons faire pour les changer. Paris, France : Éditions Les liens qui libèrent.

Stout, L. (2003). Investors' choices: The shareholder as Ulysses: Some empirical evidence on why investors in public corporations tolerate board governance. University of Pennsylvania Law Review, 152, 667-712.

Tchotourian, I. (2014). Devoir de prudence et de diligence des administrateurs et RSE : approche comparative et prospective. Cowansville, Québec : Éditions Yvon Blais.

Truffaut, S. (2017). Anatomie d’un désastre. Montréal, Québec : Éditions Somme toute.

VanDuzer, J. A. (2010). BCE v. Debentureholders : The Supreme Court's hits and misses in its most important corporate law decision since Peoples. U.B.C.L. Rev., 43, 205-258.

Von Hayek, F. A. (1989). The pretence of knowledge' prize lecture, the sveriges riksbank prize in economic science in memory of Alfred Nobel. The American Economic Review, 79(6), 3-7.

Ziegler, J. (2002). Les nouveaux maîtres du monde. Paris, France : Fayard. 